\title{
Ultrasound-Guided Hysteroscopic Removal of IUD with Missing Tails in a 15-Weeks' Pregnant Woman
}

\author{
Mustafa Albayrak, MD, Ahmet Karatas, MD, Ismail Biyik, MD, Fatih Keskin, MD \\ Department of Obstetrics and Gynecology, Duzce University School of Medicine, Duzce, Turkey (Drs Albayrak, Keskin). \\ Department of Obstetrics and Gynecology, Abant Izzet Baysal University, Bolu, Turkey (Dr Karatas). \\ Department of Obstetrics and Gynecology, Karacabey State Hospital, Karacabey, Bursa, Turkey (Dr Biyik).
}

\begin{abstract}
Unexpected pregnancy with an intrauterine device (IUD) in situ is not an uncommon finding in daily practice. It is generally agreed that an IUD should be removed in the first trimester when its tail is visible vaginally. However, because of limited experience and data in the literature, uncertainty exists about the management and technique of IUD removal during pregnancy when the tails are inaccessible on examination, especially in the second trimester. The IUD may be left in situ or removed using a grasping tool under ultrasonographic guidance or by hysteroscopy. Here, we report a case of ultrasound-guided hysteroscopic removal of an IUD with retracted tail in a woman at 15 weeks' gestation. We believe when the decision is made to remove an IUD, ultrasound-guided hysteroscopy may be a safer option in experienced hands compared with an ultrasound-guided grasping tool, especially during the second trimester.
\end{abstract}

Key Words: IUD, Hysteroscopy, Contraception, Endoscopy, Pregnancy.

Citation Albayrak M, Karatas A, Biyik I, Keskin F. Ultrasound-guided hysteroscopic removal of IUD with missing tails in a 15-weeks' pregnant woman. CRSLS e2013.00062. DOI: 10.4293/CRSLS.2013.00062.

Copyright $(0) 2015$ by SLS, Society of Laparoendoscopic Surgeons. This is an open-access article distributed under the terms of the Creative Commons Attribution-Noncommercial-ShareAlike 3.0 Unported license, which permits unrestricted noncommercial use, distribution, and reproduction in any medium, provided the original author and source are credited.

Address correspondence to: Ahmet Karatas, MD, Abant Izzet Baysal University No: Z-04 Merkez 14000 Bolu, Turkey. Telephone: +90 3742534671 , E-mail: akaratas1973@hotmail.com

\section{INTRODUCTION}

An intrauterine device (IUD) is the most frequently used reversible family planning method available worldwide. Therefore, unexpected pregnancy with an IUD in situ is not an uncommon finding in daily practice. Usually, removal of an IUD is optimal in the first trimester when tails are visible vaginally because of an $\sim 50 \%$ risk of spontaneous miscarriage, septic abortion, and preterm delivery. However, because of limited experience and data in the literature, uncertainty exists about the management and removal technique during pregnancy when tails are inaccessible on examination, especially during the second trimester. The IUD may be left in situ or removed using a grasping tool under ultrasound guidance or with hysteroscopy. ${ }^{1-4}$

We report ultrasound-guided hysteroscopic removal of an IUD with retracted tails in a woman at 15 weeks' gestation.

\section{CASE REPORT}

A 32-year-old woman (gravida 3, para 2) at 15 weeks' gestation, with a copper IUD in situ with missing tails, was referred for minimal uterine bleeding. At vaginal examination, the IUD tail was not visible and the IUD was situated at the isthmus posteriorly close to the internal cervical os and anterior and inferiorly to the gestational sac, which was located by ultrasonography at the fundus. After consulting with the family regarding the risks and benefits of either removing or leaving the IUD, they asked for its removal. With the patient under spinal anesthesia, the anterior lip of the cervix was grasped gently after swabbing the vagina with iodine and saline. Under ultrasonographic guidance and using a slow saline infusion, a 7 -mm rigid hysteroscope was easily introduced into the cervical canal without cervical dilatation. After passing the internal cervical os, the IUD was located and removed. The procedure was accomplished easily in less than 1 
minute. The patient was discharged the following day without complication. The remainder of her pregnancy was uneventful and she delivered a healthy baby at 38 weeks' gestation.

\section{DISCUSSION}

Hysteroscopic or ultrasound-guided removal of IUDs with retracted tails has been reported in only a few small case series in the literature. Assaf et al removed 46 of 52 IUDs with missing tails with carbon dioxide hysteroscopy successfully in pregnancies $<12$ weeks. ${ }^{2}$ Neis et al also used hysteroscopy to remove IUDs with missing tails successfully in 25 of 26 women, with 18 live births. ${ }^{3}$ Schiesser et al reported successful IUD removal with Holzgreve's pliers under ultrasound guidance in 81 of 82 cases of IUDs with retracted strings, with a $22 \%$ miscarriage rate and $13.5 \%$ preterm delivery rate. Only 6 of 81 women were in their second trimester, and the outcome did not differ in the first and second trimesters. ${ }^{4}$ Although Schiesser et al claimed that ultrasound-guided extraction seems more successful than hysteroscopic removal, this conclusion is based on very limited evidence.

We believe that extraction of an IUD with missing tail would be safer using the double-eye procedure (i.e., hysteroscopy combined with ultrasonography) compared with a single-eye procedure (i.e., ultrasonography with a grasping tool), especially in the early second trimester. Only a single case was reported thus far that used ultrasound-guided hysteroscopy to extract a levonorgestrel IUD with missing tail in a woman at 8 weeks' gestation. ${ }^{5}$ As far as we know, our case is the second and one of the most advanced gestations in the literature of an IUD in situ that was treated with hysteroscopy.

Experience in hysteroscopy and detailed consultation with the women are prerequisites of the procedure. We believe the most critical part in avoiding harm to the pregnancy during hysteroscopy is the time when transiting the cervical canal rather than the period where manipulations are being done to remove the IUD once inside the uterus. With hysteroscopy, special caution must be taken to move gently at the level of the internal cervical os when entering the uterine cavity, where the gestational sac is most likely to be located. Ultrasonography may help at that moment to locate both the hysteroscope and the gestational sac. We easily passed the cervical canal in the present case because the woman was multiparous. However, because IUDs are generally preferred by multipara women, this will not be a problem in most of them. In addition, modern office hysteroscopes with their smaller calibers would work better than conventional 7-mm hysteroscopes; however, we did not have such a facility. Another key point may be to keep the header of the hysteroscope in the opposite direction to the gestational sac, determined by ultrasonography during introduction to uterus, which may help to minimize the risk of harming the gestational sac. Therefore, pre- and intraoperative evaluations of the location and borders of the gestational sac with ultrasonography are important.

\section{CONCLUSION}

It is not necessary that IUDs with missing tails in pregnancy be removed, but we believe that when it is decided that one should be removed, ultrasound-guided hysteroscopy may be a safer option in experienced hands compared with an ultrasound-guided grasping tool, especially in women in their early second trimester.

\section{References:}

1. Brahmi D, Steenland MW, Renner RM, Gaffield ME, Curtis KM. Pregnancy outcomes with an IUD in situ: a systematic review. Contraception. 2012;85:131-139.

2. Assaf A, Gohar M, Saad S, el-Nashar A, Abdel Aziz A. Removal of intrauterine devices with missing tails during early pregnancy. Contraception. 1992;45:541-546.

3. Neis KJ, Brandner P, Otte C. Hysteroscopic removal of lost intra-uterine devices in pregnancy. Gynaecol Endosc. 1994;3: 233-237.

4. Schiesser M, Lapaire O, Tercanli S, Holzgreve W. Lost intrauterine devices during pregnancy: maternal and fetal outcome after ultrasoundguided extraction. An analysis of 82 cases. Ultrasound Obstet Gynecol. 2004;23:486-489.

5. McCarthy EA, Jagasia N, Maher P, Robinson M. Ultrasoundguided hysteroscopy to remove a levonorgestrel intrauterine system in early pregnancy. Contraception. 2012;86:587-590. 